\title{
Low-Frequency Fluid Waves in Fractures and Pipes
}

\author{
Valeri Korneev \\ Lawrence Berkeley National Laboratory, Berkeley, CA 94720
}

\begin{abstract}
Low-frequency analytical solutions have been obtained for phase velocities of symmetrical fluid waves within both an infinite fracture and a pipe filled with a viscous fluid. Three different fluid wave regimes can coexist in such objects, depending on the various combinations of object parameters. These regimes include Biot, Stoneley, and "narrow channel.” Equations for velocities of all these regimes have explicit forms and are verified by comparisons with exact solutions. Computations suggest that for pipes, the most common is the Biot regime, while for fractures it is the Stoneley regime. For real rock in fractures, the Biot regime exists only when fluid is in a gaseous state. The dominant role of fractures in rock permeability at field scales and the strong amplitude and frequency effects of Stoneley guided waves suggest the importance of incorporating these effects within poroelastic theories.
\end{abstract}

\section{INTRODUCTION}

We consider and compare propagation of a fundamental symmetrical mode (wave) for two model geometries: a fracture and a pipe, both infinite along the symmetry axis, filled with viscous fluid, and surrounded by an unbounded elastic medium. The importance of this fluid wave is a well established fact for boreholes (tube wave) and supported by recent numerical modeling results for fractures (Korneev et al, 2009; Feihner and Schmalholz, 2009; Derov et 
al., 2009; Bakulin et al, 2008), indicating the dominant role of this wave in wave propagation. In Korneev (2008), it was found that in fractures filled with viscous fluid, a fluid wave can propagate in both the Stoneley guided wave (Paillet and White, 1982) and the Biot slow wave (Biot, 1956ab) regimes. For both of these regimes, the fluid wave is dispersive, but its dependence on model parameters is significantly different. While in the Biot regime the dispersion exists only in viscous fluids and does not depend on wall elastic parameters, the Stoneley guided wave is dispersive, even for zero fluid viscosity, and depends on the wall's shear modulus. Relationships between these two regimes remained unclear: What are the conditions of their existence? What is the difference between fractures and pipes in fluid wave propagation? Hereafter, we refer to a channel when addressing fluid-containing parts for both geometries, which is a useful notation for discussion of similarities and differences between them. In this paper, we obtain such conditions for the abovementioned regimes and show that at a limit of small channel thickness, there also exists a diffusive narrow channel propagation regime, which differs from the Biot regime in its dependence on model parameters.

\section{THEORY FOR A FRACTURE}

\section{Previous Work}

The Stoneley guided wave, which propagates in a fluid layer bounded by elastic walls, was first obtained as a mathematical result by Krauklis (1962). Paillet and White (1982) rederived this solution when comparing waves in a borehole and its 2D analog. Their solution has an implicit form as a fundamental symmetric mode that propagates along the fracture with a velocity that approaches zero at zero frequency. Ferazzini and Aki (1987) applied this solution to explain low-frequency tremors observed before volcanic eruptions. 
Fluid-filled fracture waves also have been investigated both numerically and in laboratory studies to explain volcanic tremors and monitoring of hydraulic fracturing (Chouet, 1986, 1988; Ferrazzini et al., 1988; Tang and Cheng, 1988; Goloshubin, et al., 1994; Groenenboom and Falk, 2000; Groenenboom and van Dam, 2000; Groenenboom and Fokkema, 1998), Slow fluid waves are essential for generating tube-wave reflections from intersecting fractures (Hornby et al., 1989; Kostek et al., 1998 a, b; Derov et al., 2009; Ziatdinov et al., 2006). The high amplitudes of such waves make the solution of relevant problems rather simple, because we can ignore most other types of waves without compromising the result. The energytrapping ability of such waves illustrates the distinctive feature of fluid-filled fractures, specifically their waveguide-like capability of transporting energy. Propagation of the Stoneley guided waves in a fracture filled with viscous fluid was described in Korneev (2008). In the latest developments regarding the subject, Frehner and Schmalholz, (2009) modeled these waves for intersecting fractures using a finite-element method, and Korneev et al. (2009) compared analytical results with those obtained using OASES software.

\section{Fluid Waves in a Fracture}

Here, we consider all low-frequency symmetrical fluid waves using the results from Korneev (2008), but change some notations to make them uniform with a similar problem for a cylindrical pipe-also a subject of this paper. A symmetric model consists of the layer $-h / 2 \leq z \leq h / 2$, filled with viscous fluid between two homogeneous elastic half-spaces comprised of the same material. (We provide only the necessary expressions here; more details can be found in Korneev [2008].) The index $j=1$ indicates the parameters and fields 
related to the viscous fluid layer, while index $j=2$ indicates the values related to elastic halfspaces.

In both media, the relations between the body wave velocities and media parameters are the same: a longitudinal (P-) wave propagates with velocity

$$
V_{P j}=\sqrt{\frac{\lambda_{j}+2 \mu_{j}}{\rho_{j}}}
$$

and a shear (S-) wave with velocity

$$
V_{S j}=\sqrt{\frac{\mu_{j}}{\rho_{j}}},
$$

expressed through Lame constants $\lambda_{j}, \mu_{j}$ and density $\rho_{j}(j=1,2)$. We assume that $V_{P 2}>V_{S 2}>V_{P 1}$, which is the most common case for rocks at depth.

The linearized equation of motion for compressible viscous fluid takes the form (Korneev, 2008):

$$
\frac{\partial^{2} \mathbf{u}}{\partial t^{2}}-\frac{\eta}{\rho_{f}} \nabla^{2} \frac{\partial \mathbf{u}}{\partial t}-\frac{1}{\rho_{f}}\left(\xi-\frac{\eta}{3}\right) \nabla \nabla \cdot \frac{\partial \mathbf{u}}{\partial t}-c_{P}^{2} \nabla \nabla \cdot \mathbf{u}=0,
$$

with time $t$, fluid density $\rho_{f}=\rho_{1}$, viscosity coefficients $\eta$, $\xi$, and speed of sound $c_{p}$ for the zero-viscosity limit.

Using the time dependence of the fields in the form $\exp (-i \omega t)$, with angular frequency $\omega$, equation 3 describes the propagation of dissipating P- and S- waves with complex velocities:

$$
\begin{aligned}
& V_{\mathrm{P} 1}=\sqrt{c_{P}^{2}-i \frac{\omega}{\rho_{f}}\left(\xi+\frac{4 \eta}{3}\right)}, \\
& V_{S 1}=\sqrt{-\frac{i \omega \eta}{\rho_{f}}},
\end{aligned}
$$


and complex Lame constants:

$$
\begin{aligned}
& \lambda_{1}=c_{P}^{2} \rho_{f}-i \omega\left(\xi-\frac{2 \eta}{3}\right), \\
& \mu_{1}=-i \omega \eta .
\end{aligned}
$$

We seek a solution in the form of a surface wave with wavenumber $k_{x}=\frac{\omega}{\mathrm{v}_{f}}$, propagating along the $\mathrm{OX}$ axis with phase velocity $\mathrm{v}_{f}$.

Velocities in both media have corresponding wavenumbers:

$$
k_{P j}=\omega / V_{P j}, k_{S j}=\omega / V_{S j} . \quad(j=1,2) .
$$

Continuity conditions for two components of both stress and displacement at the boundaries $z= \pm h / 2$ lead to four linear equations for coefficients of those components. The dispersion equation for symmetric modes is obtained by finding values of $\mathrm{v}_{f}$ for which the determinant

$$
\begin{gathered}
\alpha_{s 1}\left[-(1-c)^{2} \xi_{1} \xi_{2} \alpha_{p 2} \alpha_{s 2} k_{x}^{2}-(b-c)^{2} \xi_{1} \chi_{1}-i c\left(k_{x}-a\right)\left(k_{x}-b\right) k_{x}\left(\alpha_{p 2} \chi_{1}+\xi_{1} \alpha_{s 2}\right)\right. \\
\left.+k_{x}^{2}\left(k_{x}-a c\right)^{2} \alpha_{p 2} \alpha_{s 2}+k_{x}^{2}(c a-b)^{2}\right]=0
\end{gathered}
$$

of the system is zero, where

$$
\begin{gathered}
\xi_{1}=\mathrm{i} \alpha_{P 1} \tanh \left(i \alpha_{P 1} \frac{h}{2}\right), \\
\chi_{1}=\mathrm{i} \alpha_{S 1} \operatorname{coth}\left(i \alpha_{S 1} \frac{h}{2}\right), \\
\alpha_{P j}=\sqrt{k_{P j}^{2}-h_{z}^{2}}, \quad \alpha_{s j}=\sqrt{k_{s j}^{2}-h_{z}^{2}}, \quad(j=1,2)
\end{gathered}
$$

and coefficients $a, b$, and $c$ have expressions 


$$
a=k_{x}\left(1-\frac{\mathrm{v}_{f}^{2}}{2 V_{S 1}^{2}}\right), \quad b=k_{x}\left(1-\frac{\mathrm{v}_{f}^{2}}{2 V_{S 2}^{2}}\right), \quad c=\frac{\mu_{1}}{\mu_{2}} .
$$

In equation 9, the factor in square brackets is exactly that of equation 23 from Korneev (2008). The factor $\alpha_{S 1}$ in equation 9 gives an extra root for the fluid wave propagating with the (rather small) velocity of the shear wave in viscous fluid given by equation 5 . Lowfrequency approximation means that both frequency $\omega$ and thickness $h$ are small enough to provide the conditions

$$
\left|\alpha_{P 1} \frac{h}{2}\right|<<1 \quad,\left|\alpha_{P 2} \frac{h}{2}\right|<<1 \text { and }\left|\alpha_{S 2} \frac{h}{2}\right|<<1 \text {, }
$$

which reduce the equation for the roots of the square brackets factor in equation 9 to

$$
\chi_{1}\left[\mathrm{k}_{x} h\left(1-\frac{\mathrm{v}_{f}^{2}}{V_{P 1}^{2}}\right)-\frac{\rho_{f} \mathrm{v}_{f}^{2}}{\rho_{2}\left(1-\gamma^{2}\right) V_{s 2}^{2}}\right]=2 .
$$

Approximating in Equation 15 the function 11 by the truncated Taylor series of the coth function

$$
\chi_{1} \approx \frac{2}{\mathrm{k}_{\mathrm{x}} h}\left(1-\frac{\mathrm{k}_{\mathrm{x}}^{2} h^{2}}{12} \frac{\mathrm{v}_{f}^{2}}{V_{S 1}^{2}}\right)
$$

we get the cubical polynomial equation

$$
\mathrm{v}_{f}^{3}+\frac{V_{S 0}^{3}}{V_{P}^{2}} \mathrm{v}_{f}^{2}-\frac{V_{S 0}^{3} V_{B 0}^{2}}{V_{P}^{2}+V_{B 0}^{2}}=0
$$

for determining $\mathrm{v}_{f}$.

Equation 17 contains three parameters, all of which are velocities with the forms (Korneev, 2008):

1.

$$
V_{s 0}=\left(\frac{\omega h \mu_{2}}{\rho_{f}}\left(1-\gamma^{2}\right)\right)^{\frac{1}{3}}
$$


(A low-frequency Stoneley wave velocity in a fracture filled with non-viscous fluid, where $\left.\gamma=V_{S 2} / V_{P 2}\right)$

2. $\quad V_{B 0}=V_{P 1} \sqrt{\frac{-i \omega \kappa_{f r} \rho_{f}}{\eta}}$,

(A low-frequency fluid wave velocity in a fracture with rigid walls (Biot regime), where $\kappa_{f r}=h^{2} / 12$ is fracture permeability; and

3.

$$
V_{P} \equiv V_{P 1},
$$

(a P-wave velocity in the fluid from equation 4 . Equation 17 has three roots, which can be explicitly found using the Cardano formula (Abramowitz and Stegun, 1972). Among these three roots, only one has a positive real part and therefore describes a physical wave. However, the structure of equation 17 allows for finding its asymptotic solutions in a straightforward way.

If the second term in equation 17 is much smaller than the first term, then

$$
\mathrm{v}_{f}=V_{S} \equiv V_{S 0} \sqrt[3]{\frac{V_{B 0}^{2}}{V_{P}^{2}+V_{B 0}^{2}}} .
$$

and

$$
\left|V_{S}\right|>>\left|\frac{V_{S 0}^{3}}{V_{P}^{2}}\right|
$$

which after simple algebra is equivalent to

$$
\left|V_{S}\right| \ll<\left|V_{B}\right|,
$$

If in Equation 21,

$$
\left|V_{P}^{2}\right|<<\left|V_{B}^{2}\right|, \quad \text { or } \quad 1<<\frac{\omega \kappa_{\text {fr }} \rho_{f}}{\eta}=\left(\frac{h}{S}\right)^{2},
$$


where viscous skin

$$
S=\sqrt{\frac{12 \eta}{\omega \rho_{f}}}
$$

is much smaller than fracture thickness, the elastic forces dominate viscous ones and fluid wave

$$
\mathrm{v}_{f} \approx V_{s 0}\left(1-\frac{i \omega \kappa_{f r} \rho_{f}}{3 \eta}\right)
$$

propagates in the Stoneley regime. This regime exists for "thick" fractures and/or low fluid viscosity.

If in Equation 21,

$$
\left|V_{P}^{2}\right|>>\left|V_{B 0}^{2}\right|, \quad \text { or } \quad 1>>\left(\frac{h}{S}\right)^{2},
$$

then viscous forces are taking over, and

$$
\mathrm{v}_{f} \approx V_{S} \sqrt[3]{\frac{V_{B}^{2}}{V_{P}^{2}}}=h_{3} \sqrt{-i \frac{\omega^{2} \mu_{2}}{12 \eta}\left(1-\gamma^{2}\right)},
$$

which was called a "thin" fracture regime in Korneev (2008), or a "narrow channel" regime of the fluid wave.

If in equation 17, the second term dominates over the first term, then

$$
\mathrm{v}_{f}=V_{B} \equiv \frac{V_{P} V_{B 0}}{\sqrt{V_{P}^{2}+V_{B 0}^{2}}},
$$

and such term relationships mean that

$$
\left|V_{B}\right|>>\left|\frac{V_{S 0}^{3}}{V_{P}^{2}}\right|,
$$

which is equivalent to 


$$
\left|V_{S}\right|>>\left|V_{B}\right|
$$

exactly the opposite result of inequality 23.

At low frequencies (inequalities 27) equation 29 gives

$$
\mathrm{v}_{f}=V_{B 0}=V_{P 1} \sqrt{\frac{-i \omega \kappa_{r r} \rho_{f}}{\eta}}
$$

which is a propagation in a Biot regime, when interaction of the fluid wave with the fracture walls occurs exclusively through viscous friction forces.

At high frequencies, (inequalities 23), equation 29 gives

$$
\mathrm{v}_{f} \approx V_{P},
$$

which is a propagation of body P-waves in the fluid.

Inequalities 23 and 31 mean that for any parameter set, a wave propagation regime corresponds to a regime with the slowest velocity. The regime change occurs when

$$
\left|V_{S}\right| \approx\left|V_{B}\right|
$$

Thus, when

$$
\left|V_{S}\right|>\left|V_{P}\right|
$$

the wave propagates with the velocity of P- waves in the fluid (equation 33). If both $V_{S}$ and $V_{B 0}$ are less than $\left|V_{P}\right|$ then, after substitution of expressions 21 and 29 into equation 34, we obtain

$$
\omega_{T} \approx \frac{1}{h}\left[\sqrt{\left(\frac{6 \eta}{h \rho_{f}}\right)^{2}+\left(\frac{\rho_{f}\left|V_{P}^{3}\right|}{\mu_{2}\left(1-\gamma^{2}\right)}\right)^{2}}-\frac{6 \eta}{h \rho_{f}}\right]
$$

for a transitional frequency $\omega_{T}$ that separates different propagation regimes. 
For thick fractures and small viscosities, when

$$
\left(\frac{h \rho_{f}^{2}\left|V_{P}^{3}\right|}{6 \eta \mu_{2}\left(1-\gamma^{2}\right)}\right)^{2}>>1
$$

a transition between Stoneley and Biot regimes takes place, and equation 36 becomes

$$
\omega_{T} \approx \frac{\rho_{f}\left|V_{P}^{3}\right|}{h \mu_{2}\left(1-\gamma^{2}\right)}
$$

If the inequality sign in 37 is reversed, then

$$
\omega_{T} \approx \frac{\left|\lambda_{1}^{3}\right|}{12 \eta \mu_{2}^{2}\left(1-\gamma^{2}\right)^{2}},
$$

and the transition occurs between the Biot and narrow channel regimes.

\section{NUMERICAL RESULTS FOR A FRACTURE}

Numerical study of fluid waves in a fracture involved comparison of obtained exact and asymptotic solutions for a set of media models with different parameters. The exact solution was obtained by a direct root search when left hand side of the equation 9 was computed on a dense grid for complex velocity $\mathrm{v}_{f}$. Both the real and imaginary parts varied in the range from zero to a value exceeding velocity in the fluid. The computed function was analyzed numerically always revealing the presence of two roots. One of the roots corresponded to velocity of shear waves in the fluid. The other root numerically coincided with the only physical solution of equation 17 which was computed using Cardano formulas. Among three roots of equation 17 just one has a positive real part giving only physically interpretable estimate for the exact solution of equation 9. The asymptotic solutions 21, 28 and 29 were 
compared with the exact solution. For all examples presented here, the parameters of the elastic medium are $V_{\mathrm{P} 2}=5000 \mathrm{~m} / \mathrm{s}, V_{\mathrm{S} 2}=3000 \mathrm{~m} / \mathrm{s}$. and $\rho_{2}=2.7 \mathrm{~g} / \mathrm{cm}^{3}$. Figure 1 shows real phase velocities as the functions of density. The parameters of the model are $c_{P}=1500 \mathrm{~m} / \mathrm{s}$, the viscosities $\eta=\varsigma=1 \mathrm{cP}$, frequency $20 \mathrm{~Hz}, c_{P}=1500 \mathrm{~m} / \mathrm{s}$ and the fracture thickness is $10^{-3}$ m. At low densities the exact solution follows the solution for the Biot regime, while at higher densities it follows the Stoneley regime. Note, that the transition between these two regimes corresponds to the conditions for inequalities 23 and 31. Figure 2 shows phase velocities as function of frequency for when fluid has same parameters as the "air" at atmospheric pressure, when $c_{P}=330 \mathrm{~m} / \mathrm{s}, \rho_{f}=0.0013 \mathrm{~g} / \mathrm{cm}^{3}, \eta=\varsigma=0.01 \mathrm{cP}$. Here the exact solution accurately follows the Biot regime solution. However, for 10 times thinner fracture $\left(h=10^{-4} \mathrm{~m}\right.$.) the narrow channel regime takes place at low frequencies (Figure 3). Case when air is compressed at $100 \mathrm{~atm}$ of hydrostatic pressure (approximately at $1 \mathrm{~km}$ depth) is shown on Figure 4, where $\rho_{f}=0.13 \mathrm{~g} / \mathrm{cm}^{3}$ and $h=10^{-3} \mathrm{~m}$. For this set of parameters the dispersion curve belongs to a transition between the Stoneley regime (equation 21) and the high frequency solution (equation 33). Water-filled fracture case with $c_{P}=1,500 \mathrm{~m} / \mathrm{s}$, $\rho_{f}=1.0 \mathrm{~g} / \mathrm{cm}^{3}, \eta=\varsigma=1 \mathrm{cP}$ and $h=10^{-3} \mathrm{~m}$. is shown on Figure 5. Results for oil-filled fracture where all the parameters have the same values as for Figure 6, except viscosity $\eta=\varsigma=10 \mathrm{cP}$ are shown on Figure 6. For seismic range of frequencies the solutions for both water and oil are well represented by Stoneley regime.

We will discuss these results together with similar results for a pipe which is the subject of the next section. 


\section{THEORY FOR A PIPE}

\section{Previous Work}

Fluid-filled boreholes are one of the most important signal-carrying channels in prospecting geophysics, and the literature on the properties of borehole fluid waves is quite extensive (White, 1983; Burridge et al, 1993, Schoenberg et al 1981, Cheng and Toksoz, 1981; Chang et al, 1988; Haddon, 1989, Norris, 1989, etc). Tube waves are used for permeability logging, fracture detection, reservoir monitoring, and borehole integrity testing. The similarity of Stoneley (tube) and Biot slow waves has been noted (Chang et al, 1988); Norris (1987) even described the tube wave as “a limiting case of the Biot slow wave.” In poroelastic theory (Biot, 1956b), it is assumed that on a pore scale the channel walls are rigid, and that a fluid wave in a cylindrical pipe is a core model for fluid-solid interaction. In reality, however, values of liquid rigidity are comparable with those of elastic rocks, and therefore wall-rigidity assumptions needs to be evaluated, as was done for the case of fractures in the previous section. Here we revisit the problem of low-frequency wave propagation in a cylindrical pipe filled with viscous fluid and demonstrate the existence of a "narrow channel" regime at nanoscale levels, in addition to the Biot and Stoneley low-frequency regimes.

\section{Fluid Waves in a Pipe}

We are interested in the low-frequency properties of purely symmetric fluid waves propagating along a thin cylindrical well (or a pipe) filled with viscous fluid. Outside of the cylinder with radius $\mathrm{R}$, the medium is elastic. In the following, index $j=1$ refers to fluid, while index $j=2$ denotes the elastic medium. Here, we also use notations introduced in equations $1-8$. 
It is assumed that longitudinal (P-) and shear (S-) waves in both media of the pipe satisfy welded boundary conditions at the pipe wall, providing continuity for stresses and displacements. (Mathematical formalism and the main expressions for wave propagation problems with cylindrical symmetry are presented in Appendix A.) The pure symmetry of the problem leaves only $\mathrm{m}=0$ in the field expansions. As a result, the propagation velocities $\mathrm{v}_{f}$ of the fluid waves can be found as the roots of the determinant

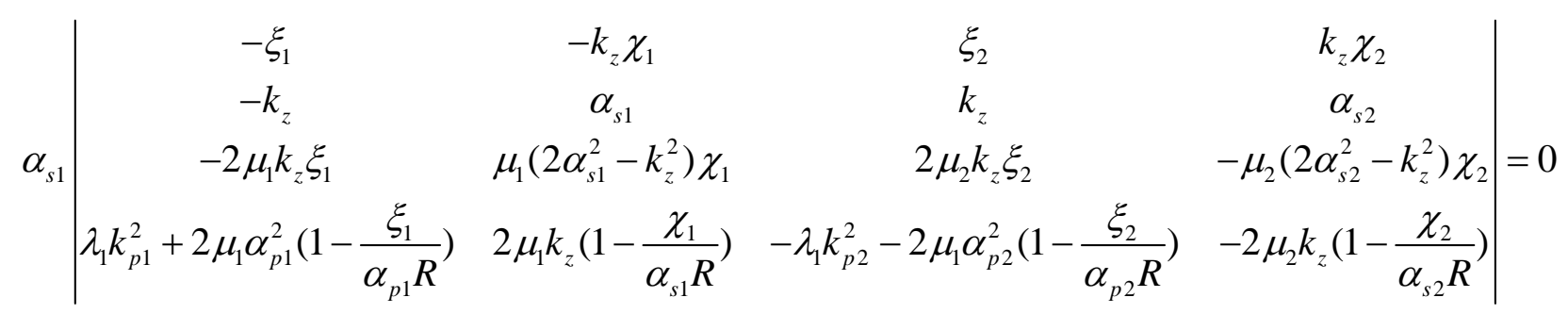

Velocity $\mathrm{v}_{f}$ is directly related to propagation wave number $k_{z}$ through the expression $\mathrm{v}_{f}=\frac{\omega}{k_{z}}$, parameters $\alpha_{P j}, \alpha_{S j}$ are defined through the formulas

$$
\alpha_{p j}=\sqrt{k_{p j}^{2}-k_{z}^{2}}, \quad \alpha_{s j}=\sqrt{k_{s j}^{2}-k_{z}^{2}} \quad j=1,2,
$$

and the functions $\xi_{1}, \chi_{1}, \xi_{2}$, and $\chi_{2}$ have the expressions

$$
\xi_{1}=\frac{J_{1}\left(\alpha_{p 1} R\right)}{J_{0}\left(\alpha_{p 1} R\right)}, \quad \chi_{1}=\frac{J_{1}\left(\alpha_{s 1} R\right)}{J_{0}\left(\alpha_{s 1} R\right)}, \quad \xi_{2}=\frac{H_{1}^{(2)}\left(\alpha_{p 2} R\right)}{H_{0}^{(2)}\left(\alpha_{p 2} R\right)}, \quad \chi_{2}=\frac{H_{1}^{(2)}\left(\alpha_{s 2} R\right)}{H_{0}^{(2)}\left(\alpha_{s 2} R\right)}
$$

where $J_{k}(Z)$ is the Bessel function, and $H_{k}^{(2)}(Z)$ is the Hankel function of the second kind.

For small arguments, $|Z|<<1$, the following asymptotic expressions

$$
\frac{J_{1}(Z)}{J_{0}(Z)} \approx \frac{Z}{2}\left(1+\frac{Z^{2}}{8}\right), \quad \frac{H_{1}^{(2)}(Z)}{H_{0}^{(2)}(Z)} \approx-\frac{1}{Z \ln (Z / 2)}
$$

can be used. 
Low-frequency assumption means that

$$
\left|\alpha_{p 1} R\right|<<1, \quad\left|\alpha_{p 2} R\right|<<1,\left|\alpha_{s 2} R\right|<<1,
$$

and the asymptotic expressions 43 can be applied to equation 40. This reduces Equation 40 to

$$
\alpha_{s 1}\left[k_{z}^{2}\left(\mu_{2} \frac{2 \chi_{1}}{\alpha_{s 1} R}-\mu_{2}\left(1+\frac{\alpha_{p 1}^{2} R^{2}}{8}\right)-\mu_{1}\right)+k_{p 1}^{2}\left(\lambda_{1}+\mu_{1}+\mu_{2}\left(1+\frac{\alpha_{p 1}^{2} R^{2}}{8}\right)\right]=0 .\right.
$$

Note that according to the equations 42 , the ratio $\frac{2 \chi_{1}}{\alpha_{s 1} R}$ from equation 45 has a limit equal to one if the argument of $\chi_{1}$ goes to zero.

Factor $\alpha_{s 1}$ gives equation 45 an immediate solution:

$$
\mathrm{v}_{f 1}=\mathrm{v}_{s 1}=\sqrt{\frac{-i \omega \eta}{\rho_{f}}}
$$

which is the same as in the fracture case.

At higher frequencies, when

$$
\left|\alpha_{s 1} R\right|>>1
$$

the argument for $\eta_{1}$ is large, and $\eta_{1} \approx i$, equation 45 becomes

$$
\frac{k_{z}^{2}}{\sqrt{k_{s 1}^{2}-k_{z}^{2}}}=i \frac{R}{2 \eta}\left[k_{p 1}^{2}\left(\lambda_{1}+\mu_{1}+\mu_{2}\right)-k_{z}^{2}\left(\mu_{1}+\mu_{2}\right)\right]=0,
$$

which can be put in a form of a cubic polynomial with respect to $k_{z}^{2}$, and solved explicitly using Cardano formulas. For the small viscosity $\eta$, the expression in square brackets must approach zero, and we obtain

$$
\mathrm{v}_{f}=\mathrm{v}_{p 1} \sqrt{\frac{\mu_{1}+\mu_{2}}{\lambda_{1}+\mu_{1}+\mu_{2}}},
$$

which for zero viscosity gives 


$$
\mathrm{v}_{f}=\mathrm{v}_{p 1} \sqrt{\frac{\mu_{2}}{\lambda_{1}+\mu_{2}}},
$$

the velocity of the Stoneley (tube) wave (White, 1965) propagating along a well filled with fluid.

When $\left|\alpha_{s 1} R\right|<<1$, then approximation 43 can be used for $\chi_{1}$, and leads to the following quadratic equation with respect to $k_{z}^{2}$

$$
k_{z}^{2}=\frac{8 k_{p 1}^{2}\left(\lambda_{1}+\mu_{1}+\mu_{2}\right)}{R^{2} \mu_{2}\left(k_{s 1}^{2}-2 k_{p 1}^{2}\right)-\mu_{1}}
$$

Introducing a dimensionless real parameter

$$
p=\frac{R^{2} \mu_{2} \rho_{f}}{8 \eta^{2}}
$$

that determines the main component in the denominator of equation 51, we find that when $p>>1$, then the module of the square of the coefficient for $k_{z}^{2}$ is much larger than the module of the last term. Thus, for $p>>1$ from equation 51 , we obtain

$$
\mathrm{v}_{f 2}=\mathrm{v}_{p 1} \sqrt{\frac{\mu_{2}}{\lambda_{1}+\mu_{2}}} \sqrt{\frac{-i \omega \kappa_{c} \rho_{f}}{\eta}},
$$

where $\kappa_{c}=\frac{R^{2}}{8}$ is pipe permeability. This wave has a velocity close to that of the Biot wave (Appendix B) for a cylinder with the "Stoneley" correction factor $\sqrt{\frac{\mu_{2}}{\lambda_{1}+\mu_{2}}}$. If the rigidity of the fluid goes to zero, then $\mathrm{v}_{f_{2}}$ (equation 53) approaches the Biot regime solution (Appendix B) for the slow wave in a pipe.

When 


$$
\sqrt{\frac{\omega \kappa_{c} \rho_{f}}{\eta}}>1
$$

the fluid wave velocity becomes undispersive and propagates as a tube wave described by equation 50.

When $p<1$ (small pipe radius), then the second term in equation 51 can be neglected, and we have

$$
\mathrm{v}_{f 2}=\mathrm{v}_{p 1} \sqrt{\frac{-i \omega \eta}{\lambda_{1}-i \omega \eta+\mu_{2}}},
$$

which describes the "narrow channel" propagation regime in the pipe.

Equations 46, 53 and 55 describe propagation of the diffusion waves.

\section{NUMERICAL RESULTS FOR A PIPE}

The main idea behind the numerical study of a pipe is the same as for the case of a fracture, which requires evaluating the validity of different propagation regimes for realistic rock parameters. The exact solution was obtained by the direct root search of equation 40 , which, similarly to the fracture case, revealed the existence of two roots. One of the roots (equation 46) corresponds to the velocity of shear waves in the fluid. The other root represents the symmetric fluid wave, which propagates in different regimes depending on parameters values. For all data presented here $c_{P}=1500 \mathrm{~m} / \mathrm{s}, \quad V_{\mathrm{P} 2}=1800 \mathrm{~m} / \mathrm{s}, \quad V_{\mathrm{S} 2}=800 \mathrm{~m} / \mathrm{s}$, $\rho_{2}=2.7 \mathrm{~g} / \mathrm{cm}^{3}$ and fluid viscosity $\eta=\varsigma=10 \mathrm{cP}$ (oil). Figure 7 shows the phase velocities for

a pipe as a function of the pipe radius $\mathrm{R}$ for $20 \mathrm{~Hz}$ frequency. Also shown are: $V_{\mathrm{P} 1}$, the solution for the Stoneley regime (equation 49), the solution for the general Biot regime (Equation 53), and the solution for "narrow channel" regime (equation 55). Figure 8 shows 
phase velocities as a function of frequency for a "thick" pipe with $10^{-4} \mathrm{~m}$ radius, while Figure 9 presents computations for a "thin" pipe with $10^{-8} \mathrm{~m}$ radius.

\section{DISCUSSION}

Theoretical and numerical results presented in this study reveal several similarities in wave propagation between fracture and pipe. The fluid symmetrical wave is always present in the solution, and it can propagate in different regimes including Stoneley, Biot and "narrow channel” regimes. The last regime occurs at very small scales, when channel width is much smaller than skin depth. This regime takes the form of equation 28 for a fracture and equation 55 for a pipe. Velocities of these waves directly depend on the shear modulus of walls, fluid viscosity, and frequency, and therefore wall elasticity is an important parameter here. These waves propagate by squeezing in fluid through the narrow channels of fractures and pipes. An extra fluid wave propagating with the velocity of a shear wave in the fluid always exists at low frequencies. It has a diffusion type of propagation and quickly dissipates. This is a generally a rather slow wave, but it can be faster than the other fluid wave, depending on the parameters.

The relationship of solutions representing both the Biot propagation regime and the Biot slow wave in poroelastic theory follows from the similarity in asymptotic expressions for these waves at low frequencies, as well as from assumptions used for deriving dynamic poroelastic equations. Section 2 of Biot (1956b) considers the oscillatory flow of fluid between two rigid parallel walls, which is the same problem for the Biot solution in an infinitely-stiff fracture considered in Korneev (2008) using a somewhat different technique. Section 3 of Biot (1956b) solves the analogous problem for a cylindrical tube (see Appendix 
C). Then, Biot calculates a friction force acting between fluid and elastic skeleton using "the assumption that the variation of friction with frequency follows the same laws as found in the foregoing for the tube of uniform cross section” (page 182). Therefore, at pore scale, the elasticity of pore walls in Biot's theory is neglected, and it operates on a macro level only, where the effects of numerous pores are evaluated. At low frequencies, the solutions for the Biot slow wave (equation C1), and Biot regimes for the fracture (equation 32) and a pipe (Equation 53) have the same dependence on frequency, permeability, and fluid viscosity, in the form

$$
V_{f}=V \sqrt{\frac{-i \omega \kappa}{\eta}}
$$

where $\mathrm{V}$ is some velocity not dependent on any parameters under the radical, $\kappa$ is permeability of the porous media, $\kappa=\frac{h^{2}}{12}$ for a fracture, and $\kappa=\frac{R^{2}}{8}$ for a pipe. However, in the two latter cases, the Biot regime transfers into narrow channel regimes at the low frequency limit.

A Stoneley wave propagation regime is very different for the considered geometries. In a pipe with a large radius (or in absence of viscosity) at low frequencies, the Stoneley wave has virtually no dispersion propagating as a tube wave. By contrast, in a fracture, the Stoneley guided wave has a strong dispersion. In a pipe, the Stoneley wave regime converts to a Biotwave regime quite rapidly, as soon as the condition

$$
\sqrt{\frac{\omega \kappa_{c} \rho_{f}}{\eta}}>1
$$

is valid. (Strictly speaking, the wave described by equation 53 is not quite a Biot wave because of the Stoneley correction factor.). In a fracture, the Biot wave regime can be 
achieved for severely contracting cases only when fluid is in a gaseous state. A quite common derivation of the Biot solution occurs when the rigidity of the walls is placed at infinity (e.g., Chang et al., 1988). Such an assumption cannot be justified from the physical point of view. Indeed, common densities and shear-wave velocities for known rocks differ from density and sound wave velocity in water by a factor of $2-3$, which is far from the high-contrast assumption between the channel walls and the fluid. Such a contrast can be achieved by applying the theory to a gas only, but this restriction is too severe for poromechanic applications.

Besides having some aforementioned similarity, the narrow channel regimes described by equations 28 and 55 differ in their dependence on fluid viscosity is different. In a fracture, this regime starts relatively quickly as the fracture thickness decreases, while for realistic parameters in pipes, it takes place surprisingly at nanoscale level at seismic frequencies. Moreover, for liquids, wall rigidity is always a factor affecting wave propagation.

The considered problems had just one fluid-channeling element, which is taken as infinite in one or two directions. Real rocks contain a wide variety of pores and fractures that can intersect, and are distributed in sizes and shapes that provide conditions for different fluid wave regimes. The fractal character of fracture and pore distribution in real rock suggests a rapid increase of their numbers for decreasing scales Diffusion waves are slow and propagate short distances, but both the numerousness of small-scale channels and reflection-refraction on heterogeneities, where diffusion waves can directly impact boundary conditions, can possibly make significant contributions to overall wave propagation. (Accurate consideration of all wave propagation effects in such media is a very complex problem, which is beyond of a scope of this study.) 
The hydrogeology results provide strong evidence that fractures play a key role in rock permeability. Permeability was measured for a wide range of scales in a number of comprehensive studies for a variety of geologic environments (Clauser, 1992; Neuman, 1994; Shultze-Makuch et al., 1999; Shultze-Makuch and Cherkauer, 1998; and Gelhar, 1993). Typically, five-orders-of-scale increase corresponds to 5-7 orders of permeability increase, suggesting the dominant role of fractures in fluid flow at field scales.

This study does not directly address the attenuation of fluid waves in channels. This is for several reasons. Such attenuation in fractures was computed and discussed in Korneev (2008) and Korneev et al. (2009). In Biot and narrow channel regimes, wave propagation describes diffusion processes that have well-known attenuation processes. General low frequency asymptotics representing solutions of cubical polynomials 17 and 39 have explicit forms and allow a simple computation of complex velocities (and, therefore, attenuation) for any chosen parameter set.

\section{CONCLUSIONS}

Analytical solutions have been obtained for the phase velocities of fluid waves within both an infinite fracture and a pipe filled with a viscous fluid at low frequencies. Two fluid waves can co-exist in such objects: diffusive wave propagating with shear-wave velocity in the fluid, and a general fluid wave that can propagate in different regimes depending on object parameters. These include Biot, Stoneley, and "narrow channel” wave regimes. Computations for realistic rock parameters suggest that for pipes, the most common regime is the Biot slow wave, while for fractures. it is the Stoneley (guided) wave. 


\section{ACKNOWLEDGMENTS}

This work was supported by the the U. S. Department of Energy under Contract No. DEAC02-05CH11231. Andrey Bakulin, Dmitry Silin, Gennady Goloshubin, German Maximov and an anonymous reviewer have made many helpful comments.

\section{REFERENCES}

Abramowitz, M. and Stegun, I. A. , 1972, Handbook of Mathematical Functions with Formulas, Graphs, and Mathematical Tables, New York: Dover.

Burridge, R., S. Kostek, and A. L. Kurkjian, 1993, Tube waves, seismic waves, and effective sources: Journal of the Acoustical Society of America,. 93, 2396-2396.

Biot, M.A., 1962, Mechanics of deformation and acoustic propagation in porous media: Journal of Applied Physics, 33, 1482-1498.

Biot, M.A., 1956a, Theory of propagation of elastic waves in a fluid-saturated porous solid. I. Low-frequency range: Journal of the Acoustical Society of America, 28, 168-178.

Biot, M.A., 1956b, Theory of propagation of elastic waves in a fluid-saturated porous solid. II. Higher frequency range: Journal of the Acoustical Society of America, 28, 179-191.

Castagna, J.P., Sun, S., and S.R. Wu., 2003, Instantaneous spectral analysis: detection of lowfrequency shadows associated with hydrocarbons: The Leading Edge, 22, 120-127.

Chang, S.K., Liu, H.L. and D.L. Johnson, 1988, Low-frequency tube waves in permeable rocks: Geophysics, 53, 519-527.

Cheng, C.H. and M.N. Toksoz, 1981, Elastic wave propagation in a fluid-filled borehole and synthetic acoustic logs: Geophysics, 46, 1042-1053. 
Chouet, B., 1988, Resonance of a fluid-driven crack: Radiation properties and implication for the source of long-period events and harmonic: Journal of Geophysical Research., 93, 43754400.

Chouet, B., 1986, Dynamics of a fluid-driven crack in three dimensions by the finitedifference method: Journal of Geophysical Research., 91,, 13967-13992.

Clauser, C., 1992, Permeability of crystalline rocks: Eos Transactions, American Geophysical Union, 73, 233.

Derov, A, Maximov, G., Lazarkov , M. , Kashtan, B., and A. Bakulin, 2009, Characterizing hydraulic fractures using slow waves in the fracture and tube waves in the borehole, 79th Annual International Meeting, SEG, Expanded Abstracts. .

Dutta, N.C. and H. Ode, 1979, Attenuation and dispersion of compressional waves in fluidfilled porous rocks with partial gas saturation (White model) - Part I: Biot theory, Geophysics, 44, 1777-1788.

Ferrazzini, V., Chouet, B., Fehler, M. and K. Aki,, 1990, Quantitative analysis of long-period events recorded during hydrofracture experiments at Fenton Hill, New Mexico: Implications for volcanic tremor: Journal of Geophysical Research, 95, 21,871-21,884.

Ferrazzini, V., and K. Aki, 1987, Slow waves trapped in a fluid-filled infinite crack: Implications for volcanic tremor, Journal of Geophysical Research, 92, 9215-9223.

Frehner, M. and S. Schmalholz, 2009, Finite-element simulations of Stoneley guided wave reflection and scattering at the tips of fluid-filled fractures: Geophysics, in print.

Gelhar, L. W., 1993, Stochastic Subsurface Hydrology, Prentice-Hall, Old Tappan, N. J. 
Goloshubin, G.M., Korneev V. A., Silin, D. B., Vingalov V.S. and C. VanSchuyer, 2006, Reservoir imaging using low frequencies of seismic reflections: The Leading Edge, 25, 527531

Goloshubin G.M., Krauklis P.V., Molotkov L.A., Helle H.B., 1994, Slow wave phenomenon at seismic frequencies: 63th Annual International Meeting, SEG, Expanded Abstracts, 809-811.

Groenenboom, J. and J. Falk, 2000, Scattering by hydraulic fractures: Finite-difference modeling and laboratory data: Geophysics, 65, 612-622.

Groenenboom, J., and D. B. van Dam, 2000, Monitoring hydraulic fracture growth: Laboratory experiments: Geophysics, 65, 603-611.

Groenenboom, J., and J. T. Fokkema, 1998, Guided waves along hydraulic fractures,: $67^{\text {th }}$ Annual SEG Meeting.

Haddon, R.A.W., 1989, Exact Green’s functions using leaking modes for axisymmetric boreholes in solid elastic media: Geophysics, 54, 609-620.

Hornby, B. E., Johnson, D. L.,Winkler, K.W., and R. A. Plumb, 1989, Fracture evaluation using reflected Stoneley-wave arrivals: Geophysics, 54, 1274-1288.

Korneev, V., A. Ponomarenko, and B. Kashtan, 2009, Stoneley guided waves: What is missing in Biot's theory?: Proceedings of the Forth Biot Conference on Poromechanics: DEStech Publication Inc., 706-711, ISBN 978-1-6059-5006-8.

Korneev, V., 2008, Slow waves in fractures filled with viscous fluid: Geophysics, 73, doi10.1190/1.2870081.

Korneev, V. A., Goloshubin, G. M., Daley, T.V., and Silin, D.B., 2004, Seismic lowfrequency effects in monitoring of fluid-saturated reservoirs, Geophysics, 69, 522-532. 
Kostek, S., Johnson, D. L., and C. J., Randall, 1998, The interaction of tube waves with borehole fractures. Part I: Numerical models: Geophysics, 63, 800-808.

Kostek, S., Johnson, D.L., Winkler, and B. E. Hornby, 1998, The interaction of tube waves with borehole fractures, Part II: Analytical models, Geophysics, 63, 809-815,

Krauklis, P. V., 1962, About some low frequency oscillations of a liquid layer in elastic medium: Prikladnaya Matematika i Mekhanika, 26, 1111-1115 (in Russian).

Landau, L.D., and E.M. Lifschitz, 1959, Fluid Mechanics: Reading, MA, Pergamon Press.

Neuman, S. P., 1994, Generalized scaling of permeabilities: Validation and effect of support scale, Geophysical Research Letters, 21, 349-352.

Norris, A., 1989, Stoneley wave attenuation and dispersion in permeable formations: Geophysics, 54, 330-341.

Norris, A., 1987, The tube wave as a Biot slow wave, : Geophysics, 52, 694-696.

Paillet, F.L. and J.E. White, 1982, Acoustic models of propagation in the borehole and their relationship to rock properties: Geophysics, 47, 1215-1228.

Schoenberg, M., T. Marzetta, J. Aron, and R.P. Porter, 1981, Space-time dependence of acoustic waves in a borehole: Journal of the Acoustical Society of America, 70, 1496-1507.

Roever, W.L., J.H. Rosenbaum, and T.F. Vining, 1974, Acoustic waves from an impulsive source in a fluid-filled borehole: Journal of the Acoustical Society of America, 55, 1144-1157.

Schulze-Makuch, D., and D. S. Cherkauer, 1998, Variations in hydraulic conductivity with scale of measurements during aquifer tests in heterogeneous, porous carbonate rock Hydrogeology Journal, 6, 204-215.

Schulze-Makuch, D., D. A. Carlson, D. S. Cherkauer, and P. Malik, 1999, Scale dependency of hydraulic conductivity in heterogeneous media: Ground Water, 37, 904-919. 
Tang, X. M. and C. H. Cheng, 1988, Wave propagation in a fluid-filled fracture - an experimental study: Geophysical Research Letters, 15, 13, 1463-1466.

White, J. E., 1965, Seismic waves: Radiation, transmission, and attenuation: McGraw-Hill

Book Co.

Ziatdinov, S., Bakulin, A. and B. Kashtan, 2006, Tube waves from a horizontal fluid-filled fracture of a finite radius: 76th Annual International Meeting, SEG, Expanded Abstracts, 369372. 


\section{FIGURES}

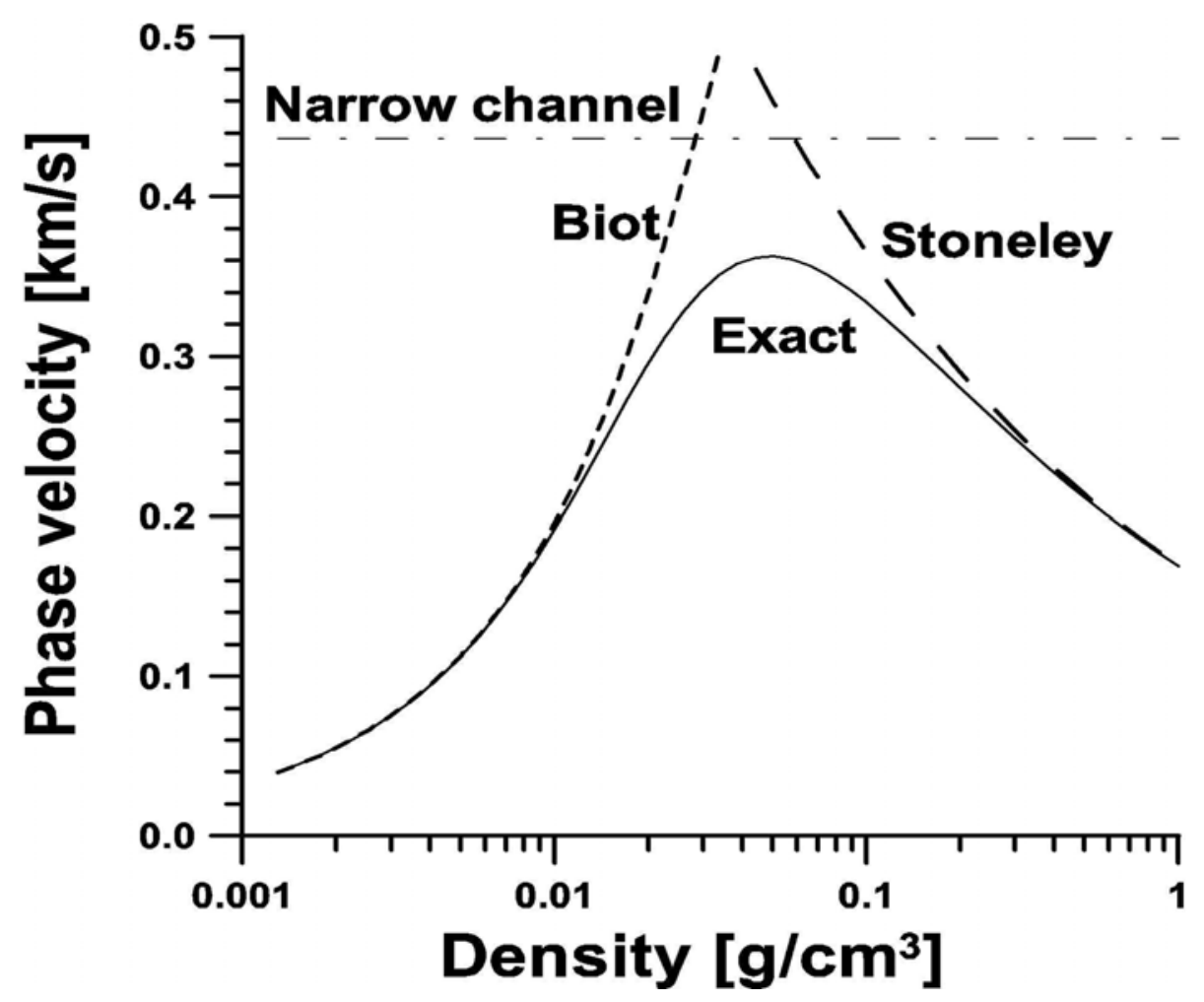

Figure 1. Phase velocity of fluid wave in a $10^{-3} \mathrm{~m}$ thick fracture as a function of fluid density. The fluid has sound velocity of $1.500 \mathrm{~m} / \mathrm{s}$ and viscosity of $1 \mathrm{cP}$, the frequency is $20 \mathrm{~Hz}$. Shown are: the exact solution (solid line) obtained from equation 9, general Biot wave regime (short dashed line) obtained from equation 29, general Stoneley wave solution (long dashed line) obtained from equation 21, and the narrow channel regime (dashed-dotted line) obtained from equation 28. Biot wave regime takes place only at very low densities. 


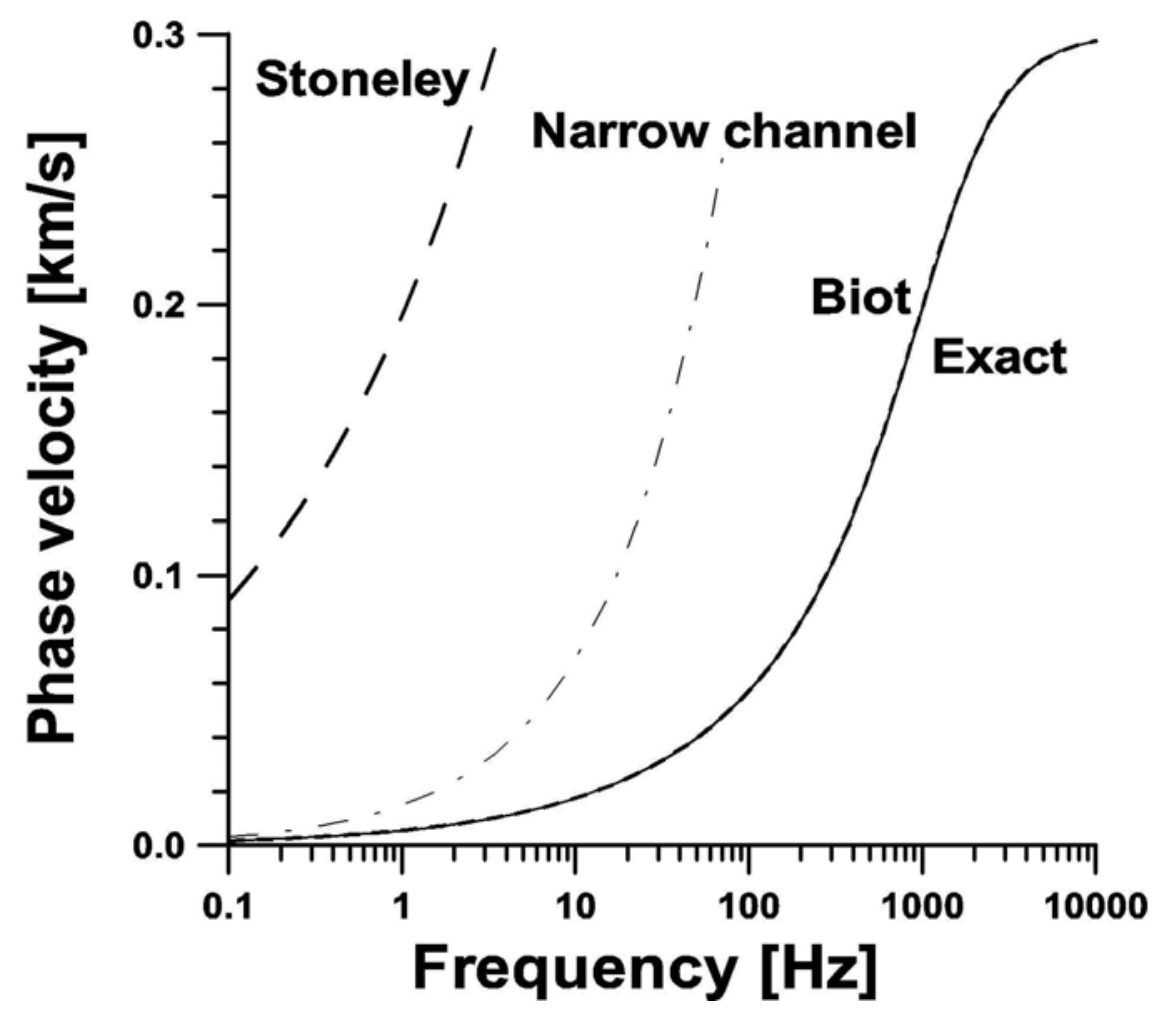

Figure 2. Phase velocity of fluid wave in a $10^{-3} \mathrm{~m}$ thick fracture as a function of frequency for the "air" at atmospheric pressure. The curves marked as on Figure 1. Biot wave solution gives accurate approximation to the exact solution. 


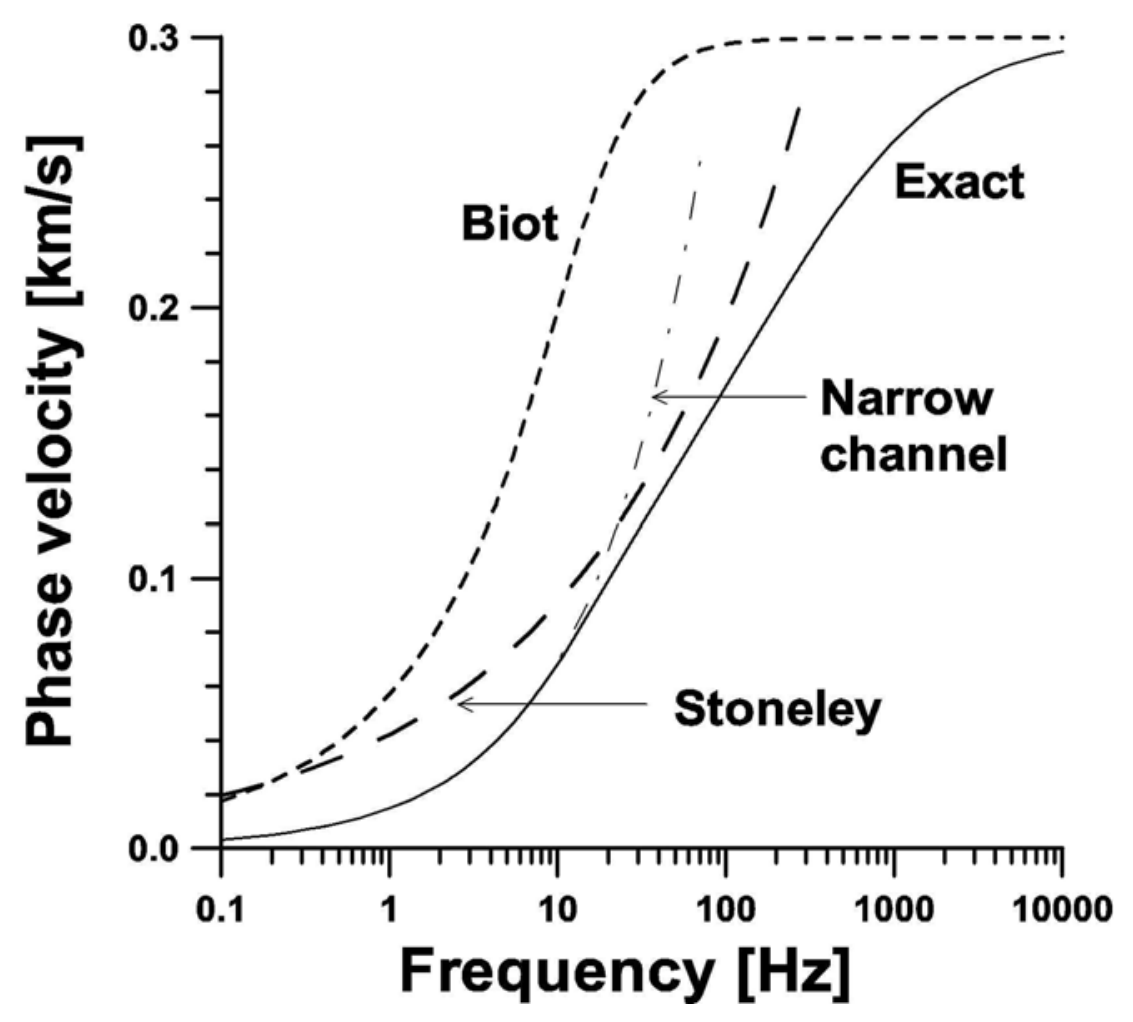

Figure 3. Phase velocity of fluid wave in a $10^{-4} \mathrm{~m}$ thick fracture as a function of frequency for the air at $100 \mathrm{~atm}(1 \mathrm{~km}$ depth) pressure. The curves marked as on Figure 1 . At low frequencies the narrow channel regime approximates the exact solution. 


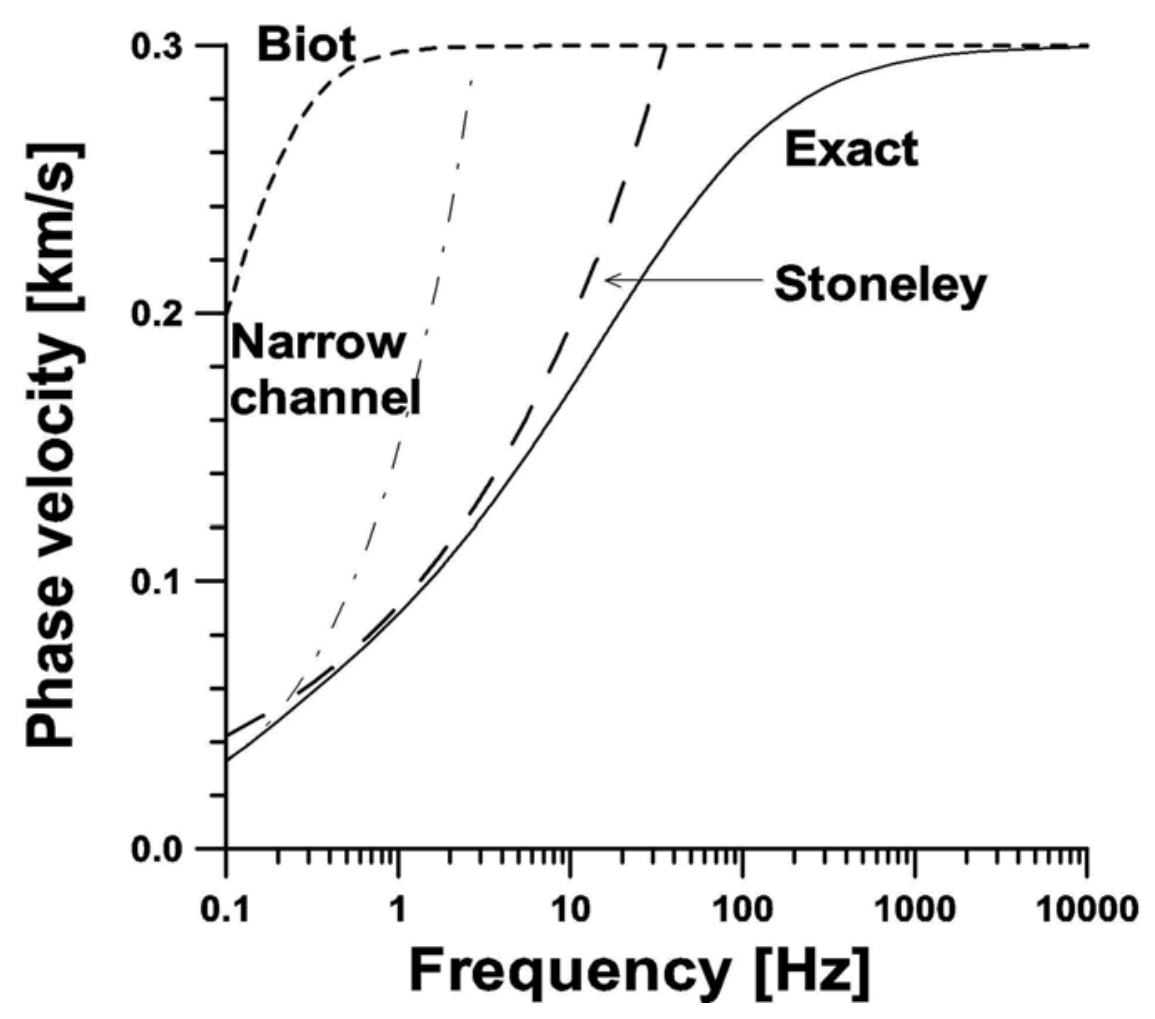

Figure 4. Phase velocity of fluid wave in a $10^{-3} \mathrm{~m}$ thick fracture as a function of frequency for the air at $100 \mathrm{~atm}(1 \mathrm{~km}$ depth) pressure. The curves marked as on Figure 1. For most frequencies the exact solution is in transitional regimes. 


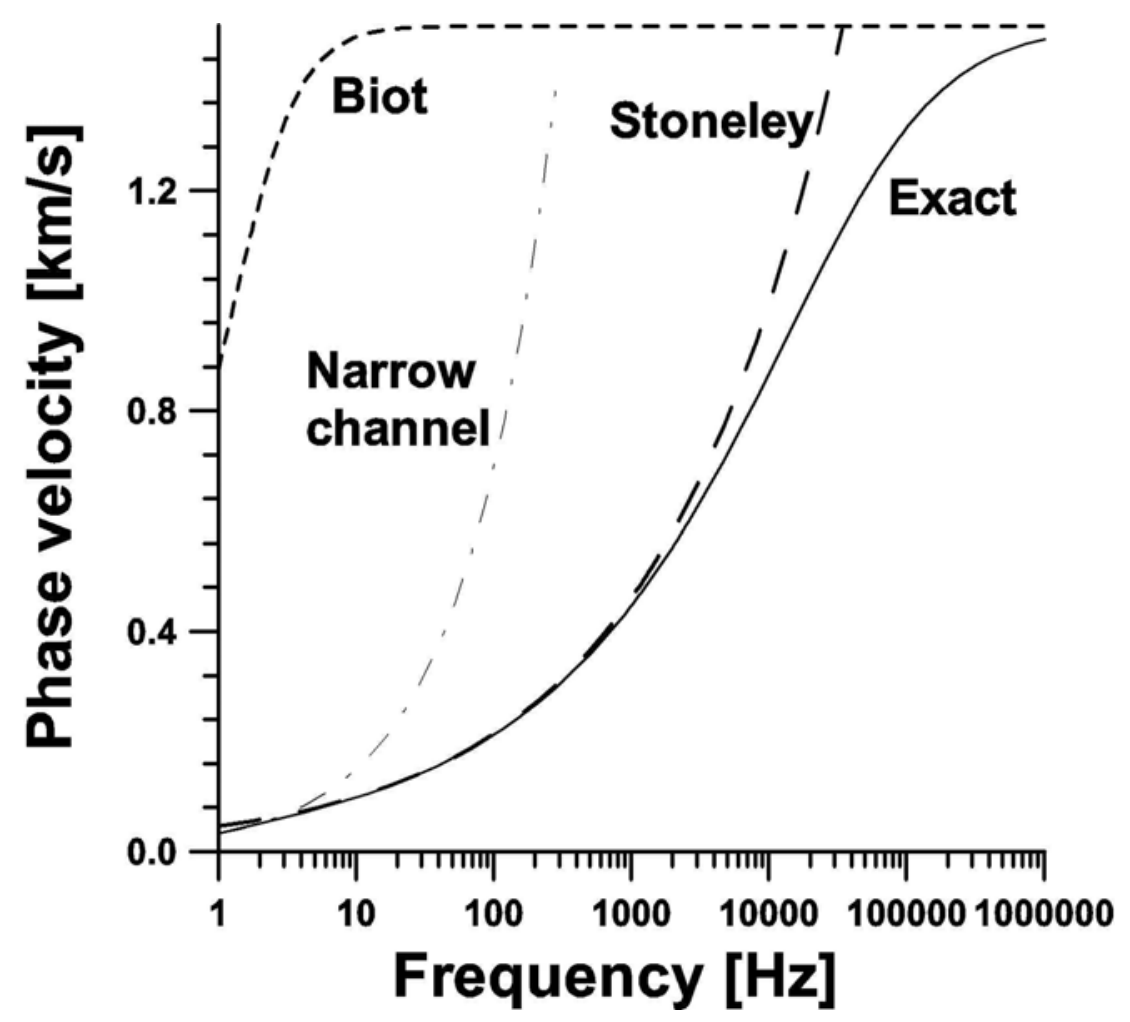

Figure 5. Phase velocity of fluid wave in a $10^{-3} \mathrm{~m}$ thick fracture as a function of frequency for water (fluid viscosity is $1 \mathrm{cP}$ ). The curves marked as on Figure 1. Stoneley wave regime approximates the exact solution at seismic frequencies. 


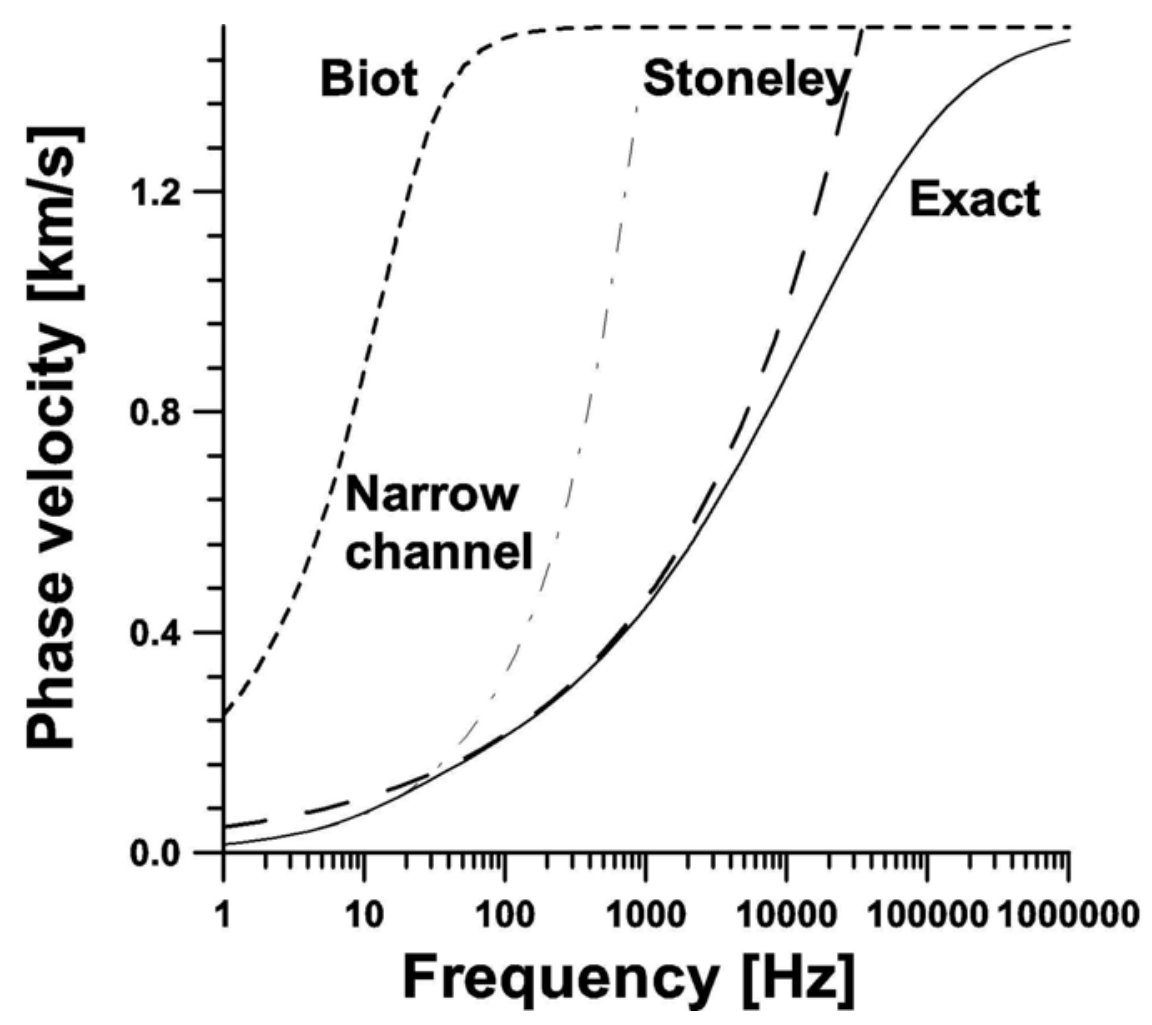

Figure 6. Phase velocity of fluid wave in a $10^{-3} \mathrm{~m}$ thick fracture as a function of frequency for the oil (fluid viscosity is $10 \mathrm{cP}$ ). The curves marked as on Figure 1 . Stoneley wave regime approximates the exact solution at seismic frequencies. 


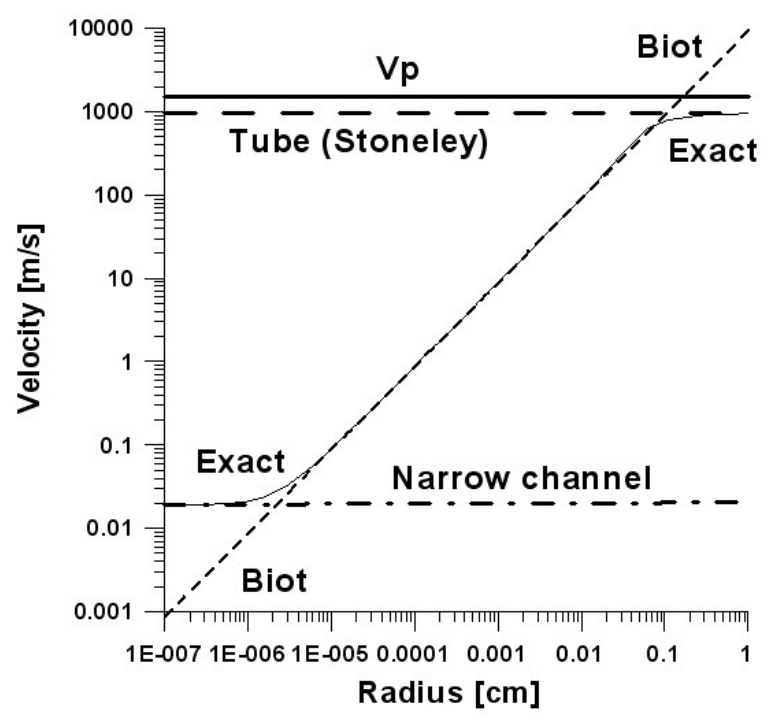

Figure 7. Phase velocity of fluid waves in a pipe filled with oil (fluid viscosity is $10 \mathrm{cP}$ ) as a function of pipe radius. Frequency is $20 \mathrm{~Hz}$. Shown are: the exact solution (thin solid line) obtained from equation 40, general Biot regime (short dashed line) obtained from equation 53, Stoneley regime (long dashed line) obtained from equation 50, P- velocity in the fluid (thick solid line, equation 4), and “narrow channel” regime (dashed-dotted line, equation 55). 


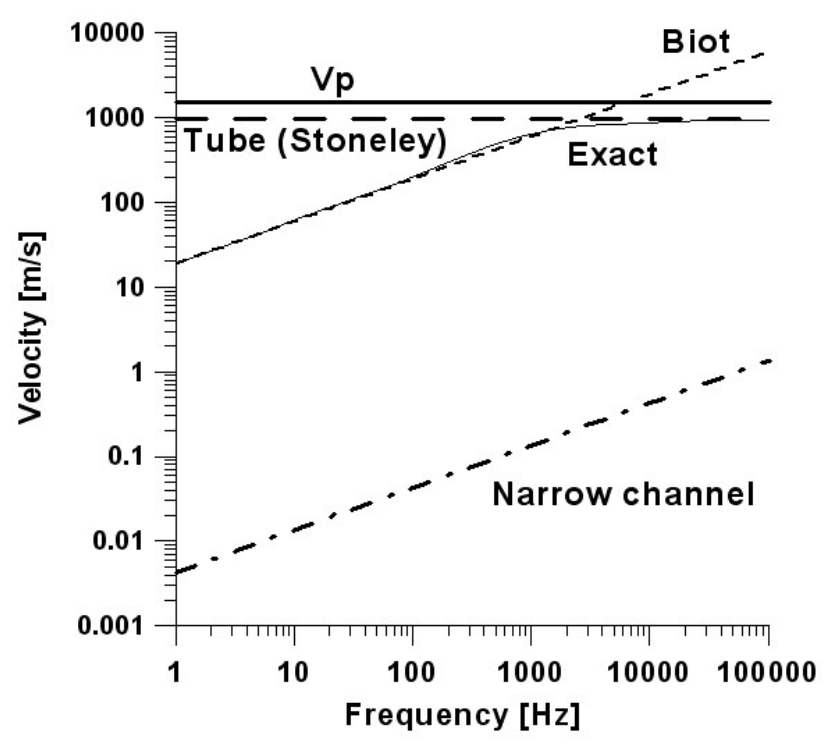

Figure 8. Phase velocity of fluid waves in a pipe filled with oil (fluid viscosity is $10 \mathrm{cP}$ ) as a function of frequency. Pipe radius is $10^{-4} \mathrm{~m}$. The curves marked as on Figure 7. 


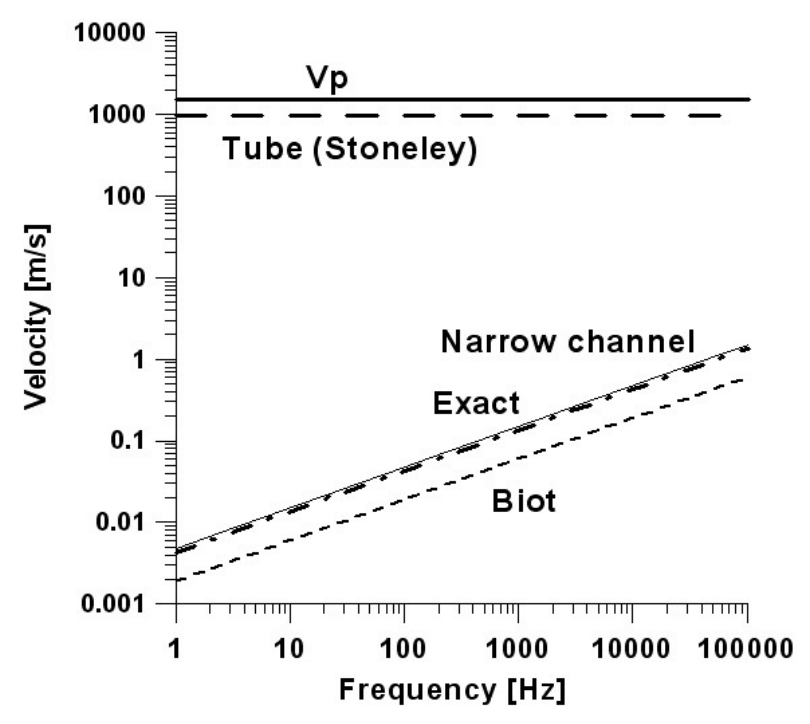

Figure 9. Phase velocity of fluid waves in a pipe filled with oil (fluid viscosity is $10 \mathrm{cP}$ ) as a function of frequency. Pipe radius is $10^{-8} \mathrm{~m}$. The curves marked as on Figure 6 . 


\section{APPENDIX A}

\section{Cylindrical vector system}

The cylindrical vector system used in this paper was introduced by Korneev and Johnson (1993). Use of those vectors makes expressions for the Lamé equation especially simple since they thoroughly imply a special symmetry of the problem. They allow formulation of wave propagation problems in displacements, avoiding the potentials.

The cylindrical vector system has the form

$$
\mathbf{Y}_{m}^{0}=Y_{m} \mathbf{e}_{3}, \quad \mathbf{Y}_{m}^{+}=\frac{1}{2}\left(Y_{m} \mathbf{e}_{1}-i Y_{m} \mathbf{e}_{2}\right), \quad \mathbf{Y}_{m}^{-}=\frac{1}{2}\left(Y_{m} \mathbf{e}_{1}+i Y_{m} \mathbf{e}_{2}\right),
$$

where

$$
Y_{m} \equiv Y_{m}(\varphi, z) \equiv \exp i\left(m \varphi-h_{z} z\right), \quad m=0,1,2, \ldots,
$$

and $h_{z}$ is the projection of the wavenumber onto the OZ-axis, $i=\sqrt{-1}$. Vectors $\mathbf{e}_{1}, \mathbf{e}_{2}, \mathbf{e}_{3}$ are the natural unit vectors of the cylindrical coordinate $\operatorname{system}(r, \varphi, z)$.

The cylindrical vectors of the system in (A1) are orthonormal at any point on a cylindrical surface. In the space of vector functions $\vec{f}(\varphi), 0 \leq \varphi \leq 2 \pi$ defined on a circle $r=$ const., $\mathrm{Z}=$ const.$\quad$ the vectors (A1) satisfy the following orthogonality relations

$$
\int_{0}^{2 \pi}\left(\mathbf{Y}_{m}^{v} \cdot \overline{\mathbf{Y}}_{m_{1}}^{v_{1}}\right) d \varphi=\delta_{m m_{1}} \delta_{v v_{1}}, \quad v=0,+,-
$$

where $\delta_{k l}$ is equal to 1 , when lower indexes are the same, and equal zero otherwise.

The system A1 is complete in the sense of convergence in the mean for a Fourier series expansion. This means that any vector function

$$
\vec{u} \equiv \vec{u}(r, \varphi, z)=\vec{U}(r, \varphi) \exp \left(-i h_{z} z\right)
$$

can be represented in the form

$$
\vec{u}(r, \varphi, z)=\sum_{v} \sum_{m=0}^{\infty} f_{m}^{v}(r) \mathbf{Y}_{m}^{v}(\varphi, z)
$$

The Lamé equation for a homogenous elastic medium is 


$$
(\lambda+\mu) \nabla \nabla \cdot \vec{u}-\mu \nabla \times \nabla \times \vec{u}+\rho \omega^{2} \vec{u}=0
$$

where the dependence of the displacement field $\vec{u}$ on time $t$ is given by $\exp (i \omega t)$, where $\omega$ is the angular frequency. The parameters $\lambda$ and $\mu$ from A7 are the Lamé constants, and $r$ is the density.

Substitution of the form (A6) into equation A7 and use of the orthogonality property A3 yields the differential Bessel equations for radial functions $f_{m}^{v} \equiv f_{m}^{v}(r)$ :

$$
f_{m q}^{0}=d_{m q}^{0} Z_{m}\left(\alpha_{q} r\right), \quad f_{m q}^{+}=d_{m q}^{+} Z_{m+1}\left(\alpha_{q} r\right), \quad f_{m q}^{-}=d_{m q}^{-} Z_{m-1}\left(\alpha_{q} r\right), \quad q=p, s,
$$

where $Z_{k}(x)$ - are the cylindrical Bessel functions of order $k$, and $d_{m q}^{v}$ are the arbitrary constants.

The parameter $\alpha_{q}$ from equation (A8) has two forms

$$
\alpha_{p}=\sqrt{k_{p}^{2}-h_{z}^{2}}, \quad \alpha_{s}=\sqrt{k_{s}^{2}-h_{z}^{2}},
$$

where $k_{p}=\omega / \mathrm{v}_{\mathrm{p}}, k_{\mathrm{s}}=\omega / \mathrm{v}_{\mathrm{s}}$

$$
\mathrm{v}_{\mathrm{p}}=\sqrt{(\lambda+2 \mu) / \rho} \text {, and } \mathrm{v}_{\mathrm{s}}=\sqrt{\mu / \rho}
$$

are the propagation velocities of compressional $\left(\vec{u}_{p}\right)$ and shear $\left(\vec{u}_{s}\right)$ field components.

The simplicity of equations A8 illustrates the main advantage of employing the cylindrical vectors of the form A1. In other systems the expressions for radial functions contain combinations of Bessel functions and their derivatives.

Introduction of functions

$$
\psi_{m p}=Y_{m}(\varphi, z) Z_{m}\left(\alpha_{p} r\right) \quad \text { and } \quad \psi_{m s}=Y_{m}(\varphi, z) Z_{m}\left(\alpha_{s} r\right)
$$

allows representation of the field $\vec{u}$ from Equation A8 in the form

$$
\vec{u}(\rho, \varphi, z)=\sum_{m=0}^{\infty}\left(a_{m} \mathbf{P}^{(m)}+b_{m} \mathbf{S}_{\mathrm{V}}^{(m)}+c_{m} \mathbf{S}_{\mathrm{H}}^{(m)}\right)
$$


containing the canonical compressional p- waves

$$
\mathbf{P}^{(m)}=-\nabla \psi_{m p}=i h_{z} Z_{m} \mathbf{Y}_{m}^{0}+\alpha_{p} Z_{m+1} \mathbf{Y}_{m}^{+}-\alpha_{p} Z_{m-1} \mathbf{Y}_{m}^{-}=\left(-\alpha_{p} Z_{m}^{\prime} \mathbf{e}_{1}-\frac{i m}{r} Z_{m} \mathbf{e}_{2}+i h_{z} Z_{m} \mathbf{e}_{3}\right) Y_{m}
$$

and two sets of shear s- waves

$$
\begin{aligned}
& \mathbf{S}_{\mathrm{V}}^{(m)}= \nabla \times \psi_{m s} \mathbf{e}_{3}=i \alpha_{s}\left(Z_{m+1} \mathbf{Y}_{m}^{+}+Z_{m-1} \mathbf{Y}_{m}^{-}\right)=\left(\frac{i m}{r} Z_{m} \mathbf{e}_{1}-\alpha_{s} Z_{m}^{\prime} \mathbf{e}_{2}\right) Y_{m}, \\
& \mathbf{S}_{\mathrm{H}}^{(m)}=\frac{1}{\alpha_{s}} \nabla \times \nabla \times \psi_{m s} \mathbf{e}_{3}=\alpha_{s} Z_{m} \mathbf{Y}_{m}^{0}+i h_{z} Z_{m+1} \mathbf{Y}_{m}^{+}-i h_{z} Z_{m-1} \mathbf{Y}_{m}^{-}= \\
&=\left(-i h_{z} Z_{m}^{\prime} \mathbf{e}_{1}+\frac{m h_{z}}{\alpha_{s} r} Z_{m} \mathbf{e}_{2}+\alpha_{s} Z_{m} \mathbf{e}_{3}\right) Y_{m}
\end{aligned}
$$

In expressions the Bessel functions and their derivatives have arguments $\alpha_{p} r$ and $\alpha_{s} r$ correspondently for compressional $\mathbf{P}^{(m)}$ and shear $\mathbf{S}_{\mathrm{V}}^{(m)}, \mathbf{S}_{\mathrm{H}}^{(m)}$ fields, which satisfy the equations

$$
\nabla \times \mathbf{P}^{(m)}=0, \quad \nabla \cdot \mathbf{S}_{\mathrm{V}}^{(m)}=\nabla \cdot \mathbf{S}_{\mathrm{H}}^{(m)}=0, \quad\left(\mathbf{S}_{\mathrm{V}}^{(m)} \cdot \mathbf{S}_{\mathrm{H}}^{(m)}\right)=0
$$

When $h=0$ the field $\mathbf{S}_{\mathrm{H}}^{(m)}$ is polarized along $\mathrm{OZ}$ axis, while polarization of $\mathbf{S}_{\mathrm{V}}^{(m)}$ is always orthogonal to this axis.

For any integer index $m=0, \pm 1, \pm 2, \ldots$ the fields $\mathbf{P}^{(m)}, \mathbf{S}_{\mathrm{V}}^{(m)}$ and $\mathbf{S}_{\mathrm{H}}^{(m)}$ satisfy equation of motion A7, and represent an independently propagating harmonic of this index.

The expressions for the traction field on a surface $r=$ const.

$$
\mathbf{t}_{r}(\vec{u})=\lambda \nabla \cdot \vec{u} \mathbf{e}_{1}+\mu\left(2 \frac{\partial \vec{u}}{\partial r}+\mathbf{e}_{1} \times \nabla \times \vec{u}\right)
$$

have forms: 


$$
\begin{gathered}
\mathbf{t}_{r}\left(\mathbf{P}^{(m)}\right)=2 i \mu \alpha_{p} h_{z} Z_{m}^{\prime} \mathbf{Y}_{m}^{0}+\left(\frac{\lambda}{2} k_{p}^{2} Z_{m}+\mu \alpha_{p}{ }^{2} Z_{m+1}^{\prime}\right) \mathbf{Y}_{m}^{+}+\left(\frac{\lambda}{2} k_{p}^{2} Z_{m}-\mu \alpha_{p}{ }^{2} Z_{m-1}^{\prime}\right) \mathbf{Y}_{m}^{-} \\
=\left[\left(\lambda k_{p}^{2} Z_{m}+\mu \alpha_{p}{ }^{2}\left(Z_{m+1}^{\prime}-Z_{m-1}^{\prime}\right)\right) \mathbf{e}_{1}-i \mu \alpha_{p}{ }^{2}\left(Z_{m+1}^{\prime}+Z_{m-1}^{\prime}\right) \mathbf{e}_{2}+2 i \mu \alpha_{p} h_{z} Z_{m}^{\prime} \mathbf{e}_{3}\right] Y_{m} \\
\mathbf{t}_{r}\left(\mathbf{S}_{\mathrm{V}}^{(m)}\right)=\mu\left(\frac{m h_{z}}{r} Z_{m} \mathbf{Y}_{m}^{0}+i \alpha_{s}^{2}\left(Z_{m+1}^{\prime}-\frac{Z_{m}}{2}\right) \mathbf{Y}_{m}^{+}+i \alpha_{s}{ }^{2}\left(Z_{m-1}^{\prime}-\frac{Z_{m}}{2}\right) \mathbf{Y}_{m}^{-}\right) \\
=\mu\left[i \alpha_{s}^{2}\left(Z_{m+1}^{\prime}+Z_{m-1}^{\prime}\right) \mathbf{e}_{1}+\alpha_{s}\left(\alpha_{s} Z_{m+1}^{\prime}-\frac{m-1}{r} Z_{m-1}\right) \mathbf{e}_{2}+\frac{m h_{z}}{r} Z_{m} \mathbf{e}_{3}\right] Y_{m} \\
\mathbf{t}_{r}\left(\mathbf{S}_{\mathrm{H}}^{(m)}\right)=\mu\left(\left(2 \alpha_{s}^{2}-k_{s}^{2}\right) Z_{m}^{\prime} \mathbf{Y}_{m}^{0}+i h_{z} \alpha_{s} Z_{m+1}^{\prime} \mathbf{Y}_{m}^{+}-i h_{z} \alpha_{s} Z_{m-1}^{\prime} \mathbf{Y}_{m}^{-}\right) \\
=\mu\left[i h_{z} \alpha_{s}\left(Z_{m+1}^{\prime}-Z_{m-1}^{\prime}\right) \mathbf{e}_{1}+h_{z} \alpha_{s}\left(Z_{m+1}^{\prime}+Z_{m-1}^{\prime}\right) \mathbf{e}_{2}+\left(2 \alpha_{s}^{2}-k_{s}^{2}\right) Z_{m}^{\prime} \mathbf{e}_{3}\right] Y_{m}
\end{gathered}
$$

\section{APPENDIX B}

\section{Biot slow wave for a pipe}

Consider equation 3 in a cylindrical coordinate system and use the rigid wall assumption. In such model, the interaction between walls and the fluid occurs through viscous friction forces. The fluid motion is mostly directed along the central axis with a parabolic distribution across the fracture, which reaches the maximum at the center line $\mathrm{z}=0$ and zero at the walls, so

$$
\mathbf{u} \approx u_{z} \mathbf{z}_{1}=u_{0}\left(R^{2}-r^{2}\right) \mathbf{z}_{1}, u_{0}=\text { const. }
$$

Introducing the total flow

$$
F=2 \pi \int_{0}^{R} u_{z} r d r
$$

across any $z=$ const, reduces equation 3 to

$$
\frac{\partial^{2} F}{\partial t^{2}}+\frac{8 \eta}{R^{2} \rho_{f}} \frac{\partial F}{\partial t}-\frac{1}{\rho_{f}}\left(\xi+\frac{4 \eta}{3}\right) \frac{\partial^{2}}{\partial z^{2}} \frac{\partial F}{\partial t}-c_{P}^{2} \frac{\partial^{2} F}{\partial z^{2}}=0
$$


Note that pipe permeability is $\kappa_{c}=R^{2} / 8$, and, therefore, it is embedded in the denominator of the second term of equation B2. (Compare to a fracture permeabilty $\kappa_{f r}=h^{2} / 12$ ). In the frequency domain, equation B3 has the solution (Korneev et al, 2004)

$$
u=\exp (i \tilde{k} x) \exp (-i \omega t), \quad \tilde{k}=k+i \alpha
$$

with the wave number $k$, the attenuation coefficient $\alpha$ and the angular frequency $\omega$. The wavenumber components have the forms

$$
\begin{aligned}
& k=\frac{\omega}{c_{P}} \sqrt{\frac{\sqrt{(1+d g)^{2}+(d+g)^{2}}+1+d g}{2\left(1+g^{2}\right)}}, \\
& \alpha=\frac{\omega}{c_{P}} \sqrt{\frac{\sqrt{(1+d g)^{2}+(d+g)^{2}}-1-d g}{2\left(1+g^{2}\right)}},
\end{aligned}
$$

where

$$
d=\frac{12 \eta}{\omega h^{2} \rho_{f}}=\frac{\eta}{\omega \kappa_{f r} \rho_{f}}, \quad g=\frac{\omega\left(\xi+\frac{4 \eta}{3}\right)}{\rho_{f} c_{P}^{2}}
$$

At low frequencies, the phase velocity $V_{\mathrm{f}}$ of the wave described by equation $\mathrm{B} 5$ has the asymptotic form

$$
V_{\mathrm{f}}=V_{\mathrm{f}}^{\text {Biot }}=c_{P} \sqrt{\frac{2 \omega \kappa_{c} \rho_{f}}{\eta}}
$$




\section{APPENDIX C}

\section{Biot slow wave for a porous medium}

Low-frequency asymptotic for the Biot slow wave can be found in the original papers (Biot, 1956a,b). Since then numerous papers dealing with Biot's poroelastic equations were published. For example, in more contemporary notations this equation can be found in Dutta and Ode (1979). Slightly changing their notations (in order to avoid interference with those used in the main text) one can obtain a low-frequency asymptotic for Biot's slow wave in the form

$$
V_{f}=\sqrt{\frac{2 D\left(2 D G^{2}-H\right)}{H}} \sqrt{\frac{-i \omega \kappa}{\eta}},
$$

where $\eta$ is a viscosity coefficient, $\omega$ is an angular frequency, $\xi$ is a permeability of the rock. Parameters

$$
\begin{aligned}
& D=\frac{K_{s}}{2}\left[G+\frac{\phi}{K_{f}}\left(K_{s}-K_{f}\right)\right]^{-1}, \\
& H=\lambda+2 \mu, \\
& G=1-\frac{K_{m}}{K_{s}} .
\end{aligned}
$$

from equation C1 depend on the bulk moduli $K_{s}, K_{f}$ and $K_{m}$ of the discrete solid grains, the fluid, and the matrix, respectively, Lame constants of the fluid-filled matrix $\lambda$ and $\mu$, and porosity $\phi$. 


\section{DISCLAIMER}

This document was prepared as an account of work sponsored by the United States Government. While this document is believed to contain correct information, neither the United States Government nor any agency thereof, nor The Regents of the University of California, nor any of their employees, makes any warranty, express or implied, or assumes any legal responsibility for the accuracy, completeness, or usefulness of any information, apparatus, product, or process disclosed, or represents that its use would not infringe privately owned rights. Reference herein to any specific commercial product, process, or service by its trade name, trademark, manufacturer, or otherwise, does not necessarily constitute or imply its endorsement, recommendation, or favoring by the United States Government or any agency thereof, or The Regents of the University of California. The views and opinions of authors expressed herein do not necessarily state or reflect those of the United States Government or any agency thereof or The Regents of the University of California.

Ernest Orlando Lawrence Berkeley National Laboratory is an equal opportunity employer. 